\title{
Recent trends in image processing and pattern recognition
}

\author{
K. C. Santosh ${ }^{1} \cdot$ Sameer K. Antani ${ }^{2}$ \\ Published online: 27 October 2020 \\ C) Springer Science+Business Media, LLC, part of Springer Nature 2020
}

The Call for Papers of the special issue was initially sent out to the participants of the 2018 conference (2nd International Conference on Recent Trends in Image Processing and Pattern Recognition). To attract high quality research articles, we also accepted papers for review from outside the conference event. Of 123 submissions, 22 papers were accepted. The acceptance rate, therefore, is just under $18 \%$.

In "Multilevel Polygonal Descriptor Matching Defined by Combining Discrete Lines and Force Histogram Concepts," authors presented a new method to describe shapes from a set of polygonal curves using a relational descriptor. In their study, relational descriptor is the main idea of the paper.

In "An Asymmetric Cryptosystem based on the Random Weighted Singular Value Decomposition and Fractional Hartley Domain," authors proposed an encryption system for double random phase encoding based on random weighted singular value decomposition and fractional Hartley transform domain. Authors claimed that the proposed cryptosystem is efficiently compared with singular value decomposition and truncated singular value decomposition.

In "Classification of Complex Environments using Pixel Level Fusion of Satellite Data," authors analyzed composite land features by fusing two original hyperspectral and multispectral datasets. In their study, the fusion image technique was found to be superior to the single original image.

In "Image Dehazing using Window-based Integrated Means Filter," authors reported that the proposed technique outperforms the state-of-the-arts in single image dehazing approaches.

In "Research on Fundus Image Registration and Fusion Method based on Nonsubsampled Contourlet and Adaptive Pulse Coupled Neural Network," authors presented a registration and fusion method of fluorescein fundus angiography image and color fundus image that combines

\section{K. C. Santosh}

santosh.kc@usd.edu

Sameer K. Antani

santani@mail.nih.gov

1 University of South Dakota, Vermillion, SD 57069, USA

2 U.S. National Library of Medicine, NIH, Bethesda, MD 20894, USA 
Nonsubsampled Contourlet (NSCT) and adaptive Pulse Coupled Neural Network (PCNN). Authors claimed that the image fusion provides an effective reference for the clinical diagnosis of fundus diseases.

In "Super Resolution of Single Depth Image based on Multi-dictionary Learning with Edge Feature Regularization," authors focused on super resolution based on multi-dictionary learning with edge regularization model. With this, the reconstructed depth images were found to be superior with respect to the state-of-art methods.

In "A Universal Foreground Segmentation Technique using Deep Neural Network," authors presented an idea of optical-flow details to make use of temporal information in deep neural network.

In 'Removal of 'Salt \& Pepper' Noise from Color Images using Adaptive Fuzzy Technique based on Histogram Estimation," authors focused on the use of processing window that is based on local noise densities using fuzzy based criterion.

In "Image Retrieval by Integrating Global Correlation of Color and Intensity Histograms with Local Texture Features," authors integrated color, intensity histograms with local state-ofthe-art texture features to perform content-based image retrieval.

In "Image-based Features for Speech Signal Classification," authors analyzed speech signal with the help of image features. Authors used the idea of computer-based image features for speech analysis.

In "Ensembling Handcrafted Features with Deep Features: An Analytical Study for Classification of Routine Colon Cancer Histopathological Nuclei Images," authors studied deep learning models to analyze medical histopathology: classification, segmentation, and detection.

In "Non-destructive and Cost-effective 3D Plant Growth Monitoring System in Outdoor Conditions," authors monitored plant growth precisely with the use of mobile phone.

In "Fusion based Feature Reinforcement Component for Remote Sensing Image Object Detection," authors employed reinforcement component (FB-FRC) to improve image classification, where two fusion strategies are proposed: a hard-fusion strategy through artificially set rules; and a soft fusion strategy by learning the fusion parameters.

In "An Improved Cuckoo Search Algorithm for Multi-level Gray-scale Image Thresholding," authors employed computationally efficient cuckoo search algorithm.

In "Image Fuzzy Enhancement Algorithm based on Contourlet Transform Domain," authors focused on enhancing globally the texture and edge of the image.

In "Pixel Encoding for Unconstrained Face Detection," authors employed handcrafted and visual features to detect human faces. Authors claimed an improvement when handcrafted and visual features are combined.

In "Data Augmentation for Handwritten Digit Recognition using Generative Adversarial Networks (GAN)," authors focused on the technique that does not require prior knowledge of the possible variabilities that exist across examples to create novel artificial examples.

In "Akin-based Orthogonal Space (AOS): A Subspace Learning Method for Face Recognition," authors reported the use of subspace learning method is efficient for human face recognition.

In "A Kernel Machine for Hidden Object-Ranking Problems (HORPs), " authors proposed a kernel machine that allows retaining item-related ordinal information while avoiding emphasizing class-related information.

In "Verification of Genuine and Forged Offline Signatures using Siamese Neural Network (SNN)," authors reported one shot learning in SNN for signature verification. 
In "Super-Resolution Quality Criterion (SRQC): A Super-Resolution Image Quality Assessment Metric," authors reported the importance of SRQC in assessing image quality. In their experiments, authors found that the SRQC is more competent in modeling the features from curvelet transform that quantifies the quality score of the super-resolved image and it outperforms the formerly reported image quality assessment metrics.

In "Ensemble based Technique for the Assessment of Fetal Health using Cardiotocograph A Case Study with Standard Feature Reduction Techniques," authors reported the use of stateof-the-art feature reduction techniques to assess fetal health using cardiotocograph.

Within the scope of image processing pattern recognition, this special issue includes multiple applications domains, such as satellite imaging, biometrics, speech processing, medical imaging, and healthcare.

Publisher's note Springer Nature remains neutral with regard to jurisdictional claims in published maps and institutional affiliations. 